\title{
Applying Particle Swarm Optimization-Base Decision Tree Classifier for Mental Illnesses
}

\author{
Elham Salehi \\ Department of Computer Engineering, Shiraz Branch, Islamic Azad University, Shiraz, Iran \\ Mahdi Mehrabi* \\ Department of Computer Engineering, Shiraz Branch, Islamic Azad University, Shiraz, Iran
}

\begin{abstract}
Background: Data mining techniques such as clustering and classification are used to explore patient's data and extract a predictive model. Medical data set are often classified by a large number of irrelevant disease measurements(features). Feature selection is one of the most common tasks which reduces the computational cost by removing insignificant features.

Method: This paper presents a graph-based Louvain algorithm for mental illness dataset clustering and a particle swarm optimization combined with a decision tree as the classifier to select the small number of an informative feature from the thousands of features were collected from health centers consist of 1060 people in two groups of 550 patients and 510 healthy.

Result: The results show that "aggression" Finding the greatest impact on the diagnosis of mental disorders has been observed in the number of 65. After that, the features such as "prisoner in the family" and "hard labor" with 63 observations had a greater impact on the disease also the third ranking "illiterate" and "elation and euphoria" had 61 and 58 observations.
\end{abstract}

Conclusions: The classification accuracy shows that the proposed method is capable of producing good results with fewer features than the original datasets.

Keywords: Mental illness, Graph clustering, Particle swarm optimization, ID3

DOI: $10.7176 /$ JIEA/9-7-03

Publication date: December $31^{\text {st }} 2019$

\section{Introduction}

1.1 Motivation

Definitions of mental illness which are referred to in academic literature include the US surgeon general's: "Mental illness is the term that refers collectively to all diagnosable mental disorders. Mental disorders are health conditions that are characterized by alterations in thinking, mood, or behavior (or some combination thereof) associated with distress and/or impaired functioning"[1].

Diagnostic and Statistical Manual of Mental Illness listed 10 types of substances that include: Alcohol, Caffeine, Cannabis, Tumors, Inhaler, Opiates, and Sleepers Anxiolytics, Stimulant-Tobacco, tobacco, and other unknown materials. The continuous use of these materials activates the brain reward system To be The feeling of pleasure from the use of substances may be so deep that a person has natural activities Neglected and neglected[2]. Substance abuse is a common problem among psychiatric patients. Studies have shown that $50 \%$ of Mental patients have substance abuse problems[3]. one of the most reason for mental illness is a drug abuse problem because it has a direct effect on the nervous system[4]. Drug abuse or alcohol abuse can increase the risk of mental illness It can worsen symptoms of a mental illness problem[5-7].A group of Italian researchers studying patients with cancer aged 18-65 Year found that anxiety is the most important factor related to mental health, which affected their quality of life[8]

Several factors affect mental disorders, some of which include:

Genetics, Drug use, Drugs, Psychotropic, Physical illnesses, Misconceptions Social and family[9-12]. Methods that analyze the relationships between the records of patients with their mental illness are often Based on statistics and questionnaires, and less than data mining and machine learning methods for this purpose Used.

Today with the increasing development of hardware, data collection and data processing is simplified more and more. Therefore, a huge amount of data is stored in various data repositories such as data files, databases, analytical databases, data warehouses. Explosive growth in data, It also requires a series of new techniques and tools that can intelligently process these data[13].

in this research, we attempt to include data on patients with neurological disorders from several clinics and discovered the knowledge of questionnaires and records with the help of data mining such as clustering and classification.

\subsection{Background}

Classification is one of the most used methods of data mining in health care that can give researchers new insights 
and can provide unprecedented insight into the wide range of biomedical data sets $[14,15]$, researchers try to use data mining to discover knowledge amounts of data of developmentally-delayed children [16]. In other case try to find variables that predicted psychiatrists' decisions [17]. Clinicians and researcher like to know the important relationship between types of psychopathology and crucial variables however it is impossible to evaluate methods of treatment without adequate methods for diagnosis, so they tend to use of data mining method that analyzes the vast data [18]. Data mining is a credible option method than statically learning approach[19].

Methods that analyze the relationships between the records of patients with their mental illness are often based on statistics and questionnaires, and less than data mining and machine learning methods. This paper is based on previous work on data mining methods and machine learning to investigate and predict mental disorders. The following section provides a brief overview of the core techniques.

1.2.1. clustering based on Louvain algorithm

A clustering algorithm divides a data set between the different group which that each group has the most similarity and least similar to other groups[20]. Combe [21], introduce a complementary measure, based on inertia and conceived to evaluate the quality of partition of elements represented in a real vector space. For detecting communities in attributed graphs, the I-Louvain algorithm was used. Their experiments show that combining the relational information with the attributes allows detecting communities efficiently.

1.2.2. classification based on PSO algorithm

The classification used to predict group membership for data instances [22]. Drydich [23] introduce a nonstructured machine-based methodology and text-mining in conversations for categorizing mental illnesses such as schizophrenia, also Sanchez [24] based on classification methods and disability characteristics The health of people is a predictive model for diagnosing the quality of life of adults with disorders Psychological, is introduced. The subjects studied in this study are 194 people with neurological disorders from the four community-based mental health agencies from the United States. Finally, the statistical results of quality The lives of these people are presented. In addition, Van der Merwe [25] show that how PSO could find the centroids of a number of clusters

\subsection{Overview}

The remainder of this paper summaries four phases: (1) the information of samples collected from the community health center. (2) In order to use data mining techniques such as clustering and classification, information has required to change to the feature vector for each sample. (3) this phase is about data preparation that involves several different stages, including the removal of overtones, deletion of features with Abandoned amount, placement of appropriate values instead of missing values, deletion of features Inefficient and irrelevant. After completing its preprocessing, clustering algorithms can be used to explore. after that, we could use of classification algorithm or machine learning algorithm. The goal of this step is to create an intelligent model for Automatic diagnosis of mental disorders. (4) The final stage is to find out the relationship between the characteristics of patients with the type of illness. It is necessary for this part to Use a strong feature selection algorithm.

Using data mining technique to create a diagnostic model disorder that helps psychiatrist to predict a type of mental disorder through patients' characteristics, such as drug use or history of illness.

\section{Instrument and tools}

In this study, Matlab 2016 used to simulate and analyze the proposed method on a dataset of mental disorder with two groups of patients and non-patients. In all experiments, the results obtained in order to Ensure an average of 15 runs is a separate algorithm. Implementation and all experiments with The use of a 5-core Intel processor with a frequency of $2.4 \mathrm{GHz}, 8 \mathrm{~GB}$ of memory and an Enterprise operating system Windows 10 Has been done.

\section{Experiment}

\section{1. methodology}

The People referring to health centers with the help of psychologist filling questionnaire consist of personal information, cause of referral, history of current illness, Functional disorder in different situation, history of smoking and alcohol, history of physical illness we selected 1060 persons with two groups of patients and healthy in the age range of consist of man and woman.

In this study, we use questioner information to build a data set of patients and non-patients, as we showed the most important features of data set for illness disorder in table 1 . The value of all features shown as a number, for example, if the value of each feature would be yes or no, we use 0 for no and 1 for yes, the feature that doesn't have any value we set -1 for that. we show the general information of mental disorder patients and healthy people in below table 2 .

\subsection{1 k-fold class validation}

in this study we use $\mathrm{k}$-fold class validation with $\mathrm{k}=10$ to assess result, this method is one of the common validation in machine learning with three steps:

1. The general dataset dived to the k subset, each subset called fold that shown as 
$\mathrm{F}_{1}, \mathrm{~F}_{2}, \ldots, \mathrm{F}_{\mathrm{I}}, \mathrm{F}_{\mathrm{K}}$

2. $\mathrm{I}=1,2, \ldots, \mathrm{k}$

- $\quad \mathrm{F}_{\mathrm{i}}$ show as a validation set and $\mathrm{k}-1$ fold stay in a training subset

- We train the proposed model with the use of validation training subset, then measured Accuracy and efficiency.

3. We estimate the accuracy and efficiency of our learning machine by calculating the average of all accuracy.

\subsubsection{Evaluation criteria}

Evaluation of the classification model is most important in classification; in this study, we use accuracy to assess the efficiency of the proposed algorithm. this method shows how many percent of dataset classification in a true way.

$$
\text { Accuracy }=\stackrel{\mathrm{TP}+\mathrm{TN}}{\mathrm{TP}+\mathrm{FP}+\mathrm{TN}+\mathrm{FN}}
$$

$\mathrm{TN}=$ Percentage of healthy samples that are properly classified

$\mathrm{TP}=$ Percentage of patient samples that are properly classified

$\mathrm{FN}=$ Percentage of mistakes that are mistaken as healthy people

$\mathrm{FP}=$ Percentage of mistakes that are mistaken as patient people parameters of the proposed algorithm are considered in the table 3.

\subsection{3 preprocessing data}

Preprocessing data is the first step in creating any model based on data mining techniques. This step is to prepare data for processing as well as improve the quality of actual data. "prepare", "normalization" and "shuffle" is done in this part.

Since the patient ID code does not play a role in the diagnostic model, the patient ID in the first column of the dataset removed. some samples of missing data are also deleted from the dataset. These samples have lost values in some of their valuable features It is specified "-1".

Min-Max normalization is a simple technique where the technique can specifically fit the data in a pre-defined boundary with a pre-defined boundary that causes the best result, so we use this method.

$$
\begin{aligned}
& \mathrm{X}_{\mathrm{f}}^{\text {new }}=\mathrm{X}_{\mathrm{f}}-\min \mathrm{f}\left(\mathrm{X}_{\mathrm{f}}\right) \\
& \quad \operatorname{Max} \mathrm{f}\left(\mathrm{X}_{\mathrm{f}}\right)_{-} \min \mathrm{f}\left(\mathrm{X}_{\mathrm{f}}\right)
\end{aligned}
$$

Where $\min _{\mathrm{f}}\left(\mathrm{x}_{\mathrm{f}}\right)=$ minimum value of the attribute

$\max _{\mathrm{f}}\left(\mathrm{x}_{\mathrm{f}}\right)=$ maximum value of attribute

$\mathrm{x}_{\mathrm{f}}=$ value of $\mathrm{f}$ for all samples in the $[0,1]$

\subsection{4. graph display of issue}

We use clustering feature to find useful feature, so we try to show the problem as a complete weighted digraph $\mathrm{G}=$ $\left(\mathrm{E}, \mathrm{F}, \mathrm{W}_{\mathrm{F}}\right)$

Where $F=\left\{F_{1}, F_{2}, \ldots, F_{N}\right\}$, that each attribute represents a node in a digraph, $E=\left\{\left(F_{i}, F_{j}\right): F_{i}, F_{j} \varepsilon F\right\}$ shows links also $\mathrm{W}_{\mathrm{F}}:\left(\mathrm{F}_{\mathrm{i}}, \mathrm{F}_{\mathrm{j}}\right) \rightarrow \mathrm{R}$ is a function that calculates the similarity between $\mathrm{F}_{\mathrm{i}}, \mathrm{F}_{\mathrm{j}}$

In this paper, we use the Pearson correlation to calculate the similarity between $\mathrm{i}$ and $\mathrm{j}$

$w_{i j}=\left|\frac{\sum_{m}\left(x_{i}-\bar{x}_{l}\right)\left(x_{j}-\bar{x}_{j}\right)}{\sqrt{\sum_{m}\left(x_{i}-\overline{x_{l}}\right)^{2}} \sqrt{\sum_{m}\left(x_{j}-\bar{x}_{j}\right)^{2}}}\right|$

Where $\mathrm{x}_{\mathrm{i}}, \mathrm{x}_{\mathrm{j}}=$ the value of $\mathrm{F}_{\mathrm{i}}, \mathrm{F}_{\mathrm{j}}$

$\bar{x}_{\mathrm{i}}, \bar{x}_{\mathrm{j}}=$ mean value for $\mathrm{F}_{\mathrm{i}}, \mathrm{F}_{\mathrm{j}}$

\subsection{5. feature clustering algorithm based on Louvain graph}

in this section we trying to put similar features in the same clusters. Clustering makes the characteristics of each cluster most similar to each other and features available different clusters have the least similarity. Distribute data in each cluster is one of the most important criteria in clustering, which has been less appreciated in previous works. in this paper we trying to solve this problem with the use of Louvain algorithm. This algorithm uses greedy methods on a matrix, to cluster vertices of the graph. Modulation function optimization is a method for recognizing communities extensive use. The modulus in the above algorithm is an optimized value between 1- and 1 It defines the density of links within societies relative to the relationships among communities. The relation below shows the modulus function: 
$Q=\frac{1}{2 m} \sum_{i j}\left[A_{i j}-\frac{k_{i} k_{j}}{2 m}\right] \delta\left(c_{i}, c_{j}\right)$

In this relation $A_{i, j}$ is the weight between two nodes $i$ and $j$. also $k_{i}$ and $k_{j}$ are sums of weight edges connected to nods $i$ and $j$ also $m$ is the sum of all edges in graph.$c_{i,} c_{j}$ are the relation between nodes $i$ and $j$. in addition $\delta$ is The simple Delta function is as follows.

$\delta_{i j}= \begin{cases}0 & \text { if } i \neq j \\ 1 & \text { if } i=j\end{cases}$

The full optimization of the modulus function is impossible for graphs with the number of nodes This optimization is performed in two steps:

- With local optimization, this algorithm looks for small groups. For I it is calculated using the below equation:

$A Q=\left[\frac{\sum_{i n}+k_{i, i n}}{2 m}-\left(\frac{\sum_{t o t}+k_{i}}{2 m}\right)^{2}\right]-\left[\frac{\sum_{i n}}{2 m}-\left(\frac{\sum_{t o t}+k_{i}}{2 m}\right)^{2}-\left(\frac{k_{i}}{2 m}\right)^{2}\right]$

$\sum$ in $=$ sum of the weight in $\mathrm{c}$

$\sum$ tot $=$ sum of the weight of edges that connected to node $\mathrm{c}$

$\mathrm{K}_{\mathrm{i}}=$ sum of the weight of $\mathrm{i}$

$\mathrm{K}_{\mathrm{i}, \mathrm{in}}=$ sum of the weight of edges between $\mathrm{i}$ and $\mathrm{c}$

$\mathrm{m}=$ sum of all edges of the graph

- Then, clustering is continuing by integrating small groups that are able to create larger groups. The

steps are repeated so that there is no change in the cluster and the cluster is not Maximum mode.

\subsubsection{Effective feature selection with the use of PSO}

After clustering Using an optimal algorithm Particle Swarm Generation The most effective features are selected. In this study, clustering Features are used to control redundancy a restoration operator is required to correct the feature is selected $\omega$ in the process of the algorithm. Which $\varphi$ is an input weight that measures the speed of a previous repetition in the current speed, also $\mathrm{C}_{1}, \mathrm{C}_{2}$ are two random values between 0 and 1 .

$x=x+v$

$v=v \times \varphi+C_{1} \times r_{1} \times($ pbest $-x)+C_{2} \times r_{2} \times($ gbest $-x)$

\section{Result}

In this research to ensure the validity of the results,10-fold validation method used. In this way, the number of training samples per fold 954 and the number of test samples are 106. The results obtained from this study, in order to ensure further, an average of 15 repetitions of the test would be done. also, we use a method based on Louvain graph in order to clustering features, as we show the result obtained from clustering in table 4. According to the great similarity between the information of individuals in the questionnaires, most samples have the same values for Their features. Therefore, the accuracy of the proposed algorithm, with the choice of features in a variety of cases, is most often $100 \%$. In our experiment, we run the proposed algorithm with a number of different features and show the percentage of different features during the process in the table 5 .

In order to examine the most commonly used features in mental illness, the number of observations is required The attribute in the feature selection is computed with different numbers. In our experiment, we have run the proposed algorithm with a number of features ranging from 1 to 73, and calculated the number of observations (frequency) in all performances for each attribute, as we showed in fig 1.

we assign a number for each attribute and see its number in repetitions. The results show that feature 6 is called "aggression" Finding the greatest impact on the diagnosis of mental disorders has been observed in the number of 65. After that, features 44 and 52 in recognition of "prisoner in the family" and "hard labor" with 63 observations had a greater impact on the disease. In rankings 4 and 5 with the number of 55 and 3 in the name of "illiterate" and "elation and euphoria" had 61 and 58 observations.

Discussion and future work

Many studies have shown that psychological disorders have a significant relationship with substance abuse Opiate and psychoactive, as well as other illnesses. These relationships are often in statistical methods Screening was obtained through questionnaires. In this research, a model for extracting this knowledge Using data mining techniques, data from the questionnaire was provided. The proposed model of the goal was to reduce the dimensions of the problem and remove features that did not play a role in the classification. And increased computational complexity.

The proposed method in this study is to diagnose patients with psychiatric disorders, we used the decision tree, PSO clustering, and optimization algorithms. some more suggestions for future work: 
Monitor the input and output parameters of all executed call and adaptive adjustments control parameters

Using different optimizing method instead of PSO and analyzing and comparing results

Using different clustering methods and comparing them with the Louvain method

Using a valid dataset and UCI to evaluate the efficiency and performance of the proposed method

In future research, you can integrate multiple databases, the number of records in the dataset Improve mental disorders and use more and more functional features.

Also in the future research, we try to train the system by the neural network instead of the three models The proposed classification, as well as the use of the genetic algorithm for the isolation of unusual samples in the dataset.

Using new data mining algorithms, new and more sophisticated systems can be found in the system Health and treatment that are able to diagnose patients with mental disorders with high precision. It is suggested to reduce the feature

Other methods of selecting features such as evolutionary differential operators and interest rates Combine with the proposed algorithm.

\section{Discussion and future work}

Many studies have shown that psychological disorders have a significant relationship with substance abuse Opiate and psychoactive, as well as other illnesses. These relationships are often in statistical methods Screening was obtained through questionnaires. In this research, a model for extracting this knowledge Using data mining techniques, data from the questionnaire was provided. The proposed model of the goal was to reduce the dimensions of the problem and remove features that did not play a role in the classification. And increased computational complexity.

- The proposed method in this study is to diagnose patients with psychiatric disorders, we used the decision tree, PSO clustering, and optimization algorithms. some more suggestions for future work

- Monitor the input and output parameters of all executed call and adaptive adjustments control parameters

- Using different optimizing method instead of PSO and analyzing and comparing results

- Using different clustering methods and comparing them with the Louvain method

- Using a valid dataset and UCI to evaluate the efficiency and performance of the proposed method

- In future research, you can integrate multiple databases, the number of records in the dataset Improve mental disorders and use more and more functional features.

- Also in the future research, we try to train the system by the neural network instead of the three models The proposed classification, as well as the use of the genetic algorithm for the isolation of unusual samples in the dataset.

- Using new data mining algorithms, new and more sophisticated systems can be found in the system Health and treatment that are able to diagnose patients with mental disorders with high precision. It is suggested to reduce the feature Other methods of selecting features such as evolutionary differential operators and interest rates Combine with the proposed algorithm.

\section{References}

1. Services, U.S.D.o.H.a.H., Mental Health: A Report of the Surgeon General. Rockville, MD: U.S. Department of Health and Human Services; Substance Abuse and Mental Health Services Administration, Center for Mental Health Services, National Institutes of Health, National Institute of Mental Health. 1999.

2. Association., A.P., Diagnostic and statistical manual of mental disorders (DSM-5®). American Psychiatric Pub. 2013.

3. Saisan, J., Melinda S., and Jeanne S., Substance Abuse and Mental Health: Overcoming Alcohol Abuse and Drug Addiction while Coping with Depression or Anxiety. 2013.

4. $\quad$ Rexer, K., Allen, H., \& Gearan, P., Data miner survey summary.Predictive Analytics World. 2011.

5. $\quad$ Craig, R.J., Counseling the alcohol and drug dependent client: Apractical approach. 2004.

6. Regier, D.A., et al., Comorbidity of mental disorders with alcohol and other drug abuse: results from the Epidemiologic Catchment Area (ECA) study. Jama, 1990. 264(19): p. 2511-2518.

7. Moore, T.H., et al., Cannabis use and risk of psychotic or affective mental health outcomes: a systematic review. The Lancet, 2007. 370(9584): p. 319-328.

8. $\quad$ Spillers, R.L., et al., Family Caregivers and Guilt in the Context of Cancer Care. Psychosomatics, 2008. 49(6): p. 511-519.

9. Bailey, A., et al., Autism as a strongly genetic disorder: evidence from a British twin study. Psychological medicine, 1995. 25(1): p. 63-77.

10. Teesson, M., et al., Alcohol-and drug-use disorders in Australia: Implications of the National Survey of 
Mental Health and Wellbeing. Australian and New Zealand Journal of Psychiatry, 2000. 34(2): p. 206213.

11. De Hert, M., et al., Physical illness in patients with severe mental disorders. I. Prevalence, impact of medications and disparities in health care. World psychiatry, 2011. 10(1): p. 52-77.

12. Vaughn, C.E. and J.P. Leff, The influence of family and social factors on the course of psychiatric illness: A comparison of schizophrenic and depressed neurotic patients. The British Journal of Psychiatry, 1976. 129(2): p. 125-137.

13. Jiawei Hon, M.K., jain Pei, data mining concepts and techniques.

14. Tomar, D. and S. Agarwal, A survey on Data Mining approaches for Healthcare. International Journal of Bio-Science and Bio-Technology, 2013. 5(5): p. 241-266.

15. Yoo, I., et al., Data mining in healthcare and biomedicine: a survey of the literature. Journal of medical systems, 2012. 36(4): p. 2431-2448.

16. Chang, C.-L., A study of applying data mining to early intervention for developmentally-delayed children. Expert Systems with Applications, 2007. 33(2): p. 407-412.

17. Baca-García, E., et al., Using data mining to explore complex clinical decisions: a study of hospitalization after a suicide attempt. Journal of Clinical Psychiatry, 2006. 67(7): p. 1124-1132.

18. Strauss, J.S., J.J. Bartko, and W.T. Carpenter, The use of clustering techniques for the classification of psychiatric patients. The British Journal of Psychiatry, 1973. 122(570): p. 531-540.

19. King, M.W. and P.A. Resick, Data mining in psychological treatment research: A primer on classification and regression trees. Journal of Consulting and Clinical Psychology, 2014. 82(5): p. 895.

20. Verma, M., et al., A comparative study of various clustering algorithms in data mining. International Journal of Engineering Research and Applications (IJERA), 2012. 2(3): p. 1379-1384.

21. Combe, D., et al. I-Louvain: An Attributed Graph Clustering Method. in Advances in Intelligent Data Analysis XIV. 2015. Cham: Springer International Publishing.

22. Phyu, T.N. Survey of classification techniques in data mining. in Proceedings of the International MultiConference of Engineers and Computer Scientists. 2009.

23. Diederich, J., A. Al-Ajmi, and P. Yellowlees, Ex-ray: Data mining and mental health. Applied Soft Computing, 2007. 7(3): p. 923-928.

24. Sánchez, J., Rosenthal, D. A., Tansey, T. N., Frain, M. P., \& Bezyak, J. L., Predicting quality of life in adults with severe mental illness: Extendingthe International Classification of Functioning, Disability, and Health.Rehabilitation psychology. 2016.

25. Van der Merwe, D. and A.P. Engelbrecht. Data clustering using particle swarm optimization. in Evolutionary Computation, 2003. CEC'03. The 2003 Congress on. 2003. IEEE.

Notes

Table 1. Most important features of the dataset for illness disorder

\begin{tabular}{|c|c|c|c|c|}
\hline Volubility & Elation/euphoria & Over activity & Education & Id number \\
\hline Desire & Mental retardation & $\begin{array}{l}\text { Physical } \\
\text { aggression }\end{array}$ & Worthless & Divorce or death of the family \\
\hline Despair & Suicidal attempt & Suicidal ideation & Self-talk & Psychomotor retardation \\
\hline Anhedonia & $\begin{array}{l}\text { Concentration } \\
\text { disorder }\end{array}$ & $\begin{array}{l}\text { Verbal } \\
\text { Aggression }\end{array}$ & Disorientation & Chronic physical illness \\
\hline Irritability & Neglect cleaning & Memory disorder & Clapping & Underinsurance coverage \\
\hline Gender & Number of children & Hypersomnia & Sample class & Physical complaint \\
\hline Self-harm & Family disorder & $\begin{array}{l}\text { Addicted } \\
\text { family }\end{array}$ & Hyperplasia & Generalized anxiety disorder \\
\hline Sadness & Personality disorder & Hallucination & Delusions & Obsessive-compulsive disorder \\
\hline Fever & Baby jaundice & Social disorder & Job disorder & Education disorder \\
\hline Age & Family issues & $\begin{array}{l}\text { Financial } \\
\text { problems }\end{array}$ & Hard labor & $\begin{array}{l}\text { Substance and alcohol abuse } \\
\text { disorder }\end{array}$ \\
\hline Jobless & Domestic violence & Prisoner in family & Anorexia & Mental and physical retardation \\
\hline Epilepsy & Depression disorder & Anxiety disorder & Illiterate & Psychotic disorder \\
\hline Addict & No disturbance & Head of the family & Marital status & Extraordinary behavior \\
\hline Guilt & Employment & $\begin{array}{l}\text { How to start the } \\
\text { disease }\end{array}$ & $\begin{array}{l}\text { Neglect } \\
\text { disease }\end{array}$ & Child and adolescent disorder \\
\hline Insomnia & Lack of energy & Expert advisor & Incoherence & Psychomotor agitation \\
\hline
\end{tabular}


Table 2. The general information of patients and healthy people

\begin{tabular}{|l|l|l|l|l|}
\hline Class & Features & Healthy & Patients & Total samples of the dataset \\
\hline 2 & 74 & 510 & 550 & 1060 \\
\hline
\end{tabular}

Table 3. parameters of the proposed algorithm

\begin{tabular}{|c|c|c|c|}
\hline Parameter value & Parameter name & Parameter value & Parameter name \\
\hline 35 & Number of particles & 0.4 & $\theta$ \\
\hline 100 & Number of repetition & 3 & $\omega$ \\
\hline
\end{tabular}

Table 4. The result of clustering with the use of Louvain algorithm

Features

Number Cluster

of

features

How start disease, irritability, concentration disorder, self-talk, suicidal ideation, lack of energy, feeling worthless, desire, anhedonia, guilty, psychomotor retardation, physical complaints, insomnia, clapping, sadness, anorexia, generalized anxiety disorder, despair, obsessive-compulsive disorder, family disorder, personality disorder, social disorder, baby jaundice, chronic physical disorder, family issue, divorce or death of family, mental and physical retardation, aggression, anxiety disorder, depression disorder, no disturbance, head of the family, under insurance coverage, expert advisor, addict

Extraordinary behavior, neglect cleaning, neglect disease, hypersomnia, disorientation, psychotic disorder

Volubility, Hyperplasia, financial problems, addicted to family

Elation and euphoria, delusions

Hallucination, job disorder, substance and alcohol abuse disorder

Hard labor

Incoherence, fever, jobless, mental retardation

Prisoner in family

Illiterate

Suicidal attempt, self-harm, epilepsy

Over activity, physical aggression, psychomotor agitation, education disorder, child and adolescent disorder, gender, age, marital status, employment, number of children, education

Aggression

Table 5. The result of feature selection with a different number

\begin{tabular}{lll}
\hline $\begin{array}{l}\text { Precision } \\
\mathbf{( \% )}\end{array}$ & Features & $\begin{array}{c}\text { Number } \\
\text { Features }\end{array}$ \\
\hline $\mathbf{6 9}$ & How to start disease & 1 \\
\hline $\mathbf{9 2}$ & Psychomotor agitation, anorexia, & 2 \\
\hline $\mathbf{9 4}$ & Extraordinary behavior, concentration disorder, generalized anxiety disorder & 3 \\
\hline $\mathbf{1 0 0}$ & Personality disorder, epilepsy, child and adolescent disorder, addict & 4 \\
\hline $\mathbf{9 5}$ & $\begin{array}{l}\text { Elation and euphoria, memory disorder, anhedonia, psychomotor retardation, } \\
\text { Hyperphagia }\end{array}$ & 5 \\
\hline $\mathbf{9 6}$ & $\begin{array}{l}\text { Volubility, memory disorder, self-talk, psychomotor retardation, chronic physical } \\
\text { illness, a prisoner in the family }\end{array}$ & 6 \\
\hline $\mathbf{1 0 0}$ & $\begin{array}{l}\text { Self-talk, generalized anxiety disorder, education disorder, hard labor, illiterate, } \\
\text { substance and alcohol abuse disorder, no disturbance }\end{array}$ & 7 \\
\hline $\mathbf{1 0 0}$ & $\begin{array}{l}\text { How start disease, Extraordinary behavior, feeling worthless, disorientation, } \\
\text { hallucination, family issue, financial problems, family disorder }\end{array}$ & 8 \\
\hline $\mathbf{1 0 0}$ & $\begin{array}{l}\text { psychomotor retardation, hypersomnia, Hyperphagia, despair, personality } \\
\text { disorder, delusions, psychotic disorder, depression disorder, child and adolescent } \\
\text { disorder }\end{array}$ & \\
\hline $\mathbf{1 0 0}$ & $\begin{array}{l}\text { Self-talk, lack of energy, anhedonia, Psychomotor agitation, clapping, personality } \\
\text { disorder, job disorder, social disorder, , chronic physical illness, child and } \\
\text { adolescent disorder }\end{array}$ & \\
\hline
\end{tabular}




\begin{tabular}{lll}
\hline $\begin{array}{l}\text { Precision } \\
(\mathbf{\%})\end{array}$ & Features & $\begin{array}{l}\text { Number of } \\
\text { Features }\end{array}$ \\
\hline $\mathbf{1 0 0}$ & $\begin{array}{l}\text { Lack of energy, desire, physical complaints, sadness, delusions, chronic physical } \\
\text { illness, addicted in the family, illiterate, mental retardation, no disturbance, } \\
\text { employment }\end{array}$ & \\
\hline $\mathbf{1 0 0}$ & $\begin{array}{l}\text { aggression, memory disorder, self-harm, Lack of energy, feeling worthless, } \\
\text { psychomotor retardation, despair, personality disorder, family issue, depression } \\
\text { disorder, child and adolescent disorder, addict }\end{array}$ & 12 \\
\hline $\mathbf{1 0 0}$ & $\begin{array}{l}\text { Elation and euphoria, aggression, self-harm, Lack of energy, neglect cleaning, } \\
\text { Psychomotor agitation, Hyperphagia, personality disorder, hard labor, fever, the } \\
\text { prisoner in family, illiterate, psychotic disorder, substance and alcohol abuse } \\
\text { disorder }\end{array}$ & \\
\hline $\mathbf{1 0 0}$ & $\begin{array}{l}\text { Overactivity, elation and euphoria, aggression, suicidal attempt, neglect disease, 14 } \\
\text { hypersomnia, hallucination, hard labor, fever, addicted in the family, a prisoner in } \\
\text { the family, illiterate, no disturbance, head of the family }\end{array}$ \\
\hline $\mathbf{1 0 0}$ & $\begin{array}{l}\text { Elation and euphoria, volubility, aggression, physical aggression, suicidal attempt, } \\
\text { lack of energy, neglected disease, anorexia, hallucination, hard labor, fever, } \\
\text { prisoner in family, illiterate, psychotic disease, no disturbance }\end{array}$ \\
\hline
\end{tabular}

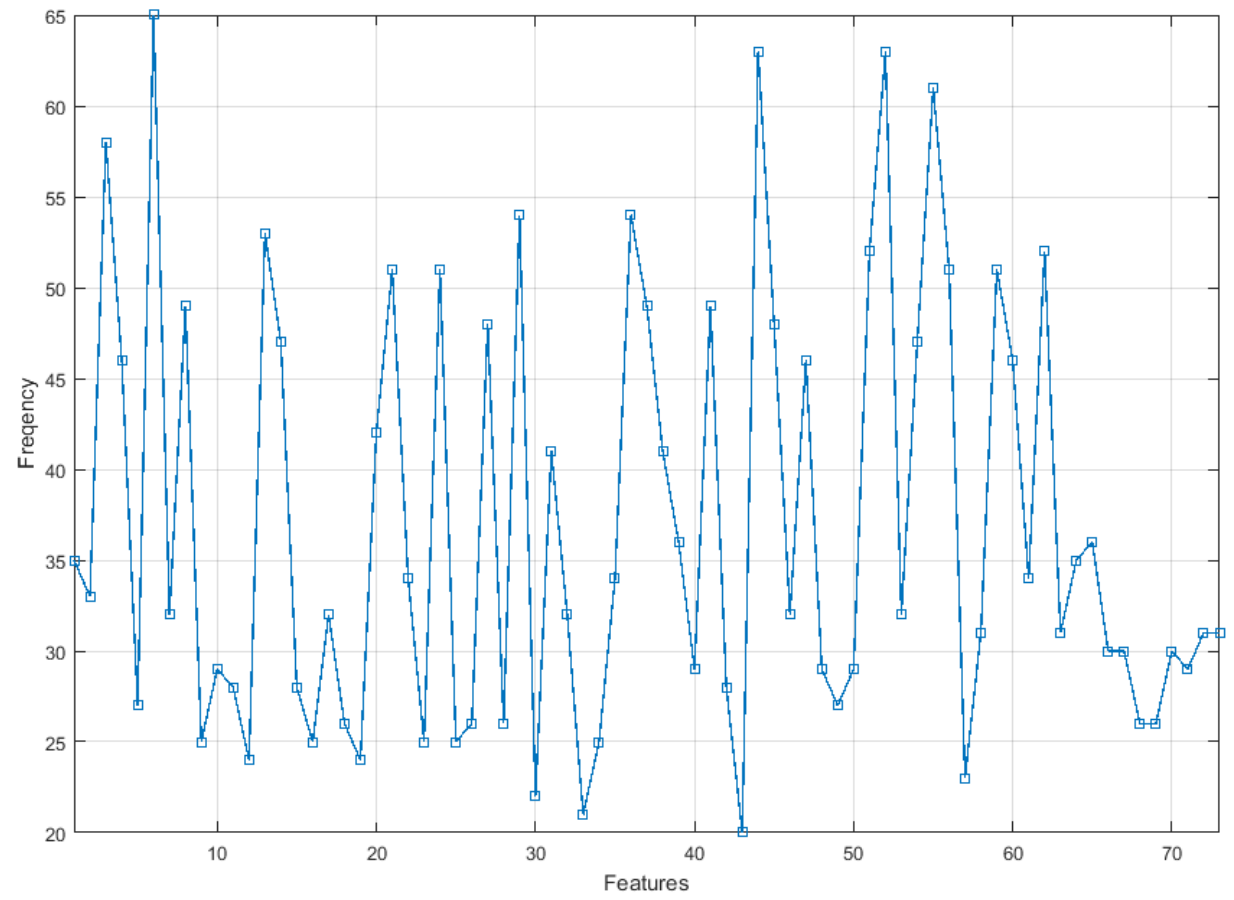

Fig1. The frequency of repeating each attribute in the data of mental disorders 